\title{
Identification of SiC Crystals Based on 3D Reconstruction Using EBSD Technique
}

\author{
Ming Han ${ }^{1}$, Guangming Zhao ${ }^{1}$
}

${ }^{1 .}$ School of Materials Science and Engineering, East China Jiaotong University, Jiangxi, China.

Almost all applications of electron backscatter diffraction (EBSD) technique for the present are based on accurate determination of the crystal orientation. Only after correctly indexing the lattice planes $(h k l)$ of the Kikuchi bands and the lattice directions $[u v w]$ of the Kikuchi poles in an EBSD pattern, the Euler angles representing the crystal orientation can be derived. For the crystals with various polytypes, however, their pattern indexing is not a simple work to accomplish. For example, silicon carbide (SiC) had been identified to have more than 250 polytypes [1], some of which have a lattice constant as long as $301.5 \mathrm{~nm}$, about one thousand times the usual $\mathrm{SiC}$ lattice spacings [2]. Besides the cubic structure, $\mathrm{SiC}$ also has plenty of hexagonal and rhombohedral structures. The lattice constant $a$ of different SiC hexagonal structures are almost equal. In real space, longer lattice constant $c$ corresponds to a shorter value of $c^{*}$ axis in reciprocal space, which means that the $d$-spacings of the lattice planes $(h k l)$ and $(h k$ $l \pm 1$ ) are almost the same for some SiC crystals with long $c$ axis. Therefore, indexing the diffraction patterns of unusual SiC crystals may be extremely difficult. A clear detection and good match of only a few Kikuchi bands does not guarantee a correct indexing and usually the indexed results incline to be erroneous.

In this paper, we apply a three-dimensional reconstruction method to some EBSD patterns of different $\mathrm{SiC}$ crystals, use the recognizable Kikuchi bands and Kikuchi poles in the patterns, and directly identify the Bravais lattices of the crystals via geometric reconstruction. The Miller indices of Kikuchi bands and Kikuchi poles can also be easily indexed based on the determination result.

In Figure 1a, the traces (blue lines) of diffracting lattice planes are interconnected to each other. The four circles mark the Kikuchi poles (zone axes) which are used to align the positions of all traces and zone axes. This alignment process may include many times of small positional refinement with the criterion that the calculated hyperbolae should match the edges of Kikuchi bands as close as possible. The positions of zone axes and the source of the EBSD pattern influence the axial ratio of the determined Bravais lattice. Theoretically, by using the width of only one Kikuchi band, the absolute values of lattice constants can be calculated. Hence the Kikuchi bands with clear edge contrast and wide widths are beneficial to increasing the accuracy of the lattice constants. In Figure 1, the red hyperbolae and corresponding Kikuchi bands are chosen to determine the absolute values of the lattice constants. The reasonableness of final identification results can be judged based on a criterion, i.e., all the calculated hyperbolae must match well with the edge of visible Kikuchi bands. For the pattern in Figure 1a, the identification result is a hexagonal Bravais lattice with $a=0.3035 \mathrm{~nm}$ and $c=1.5019 \mathrm{~nm}(6 \mathrm{H})$, which is in good agreement with the literatures [3, 4]. For the pattern in Figure 1b, the identification result is a rhombohedral lattice with $a=1.2776 \mathrm{~nm}, \alpha=13.85^{\circ}$. This rhombohedral lattice can also be described by a hexagonal unit cell with $a=0.3080 \mathrm{~nm}, c=3.7954 \mathrm{~nm}$, which agree well the reported 15R structure [5] [6].

References: 
[1] R. Cheung, in "Silicon Carbide Microelectromechanical Systems for Harsh Environments", R. Cheung, (Imperial College Press) p.3.

[2] J.F. Kelly, G.R. Fisher, P. Barnes, Mater. Res. Bull. 40 (2005), p. 249.

[3] G.C. Capitani, S.D. Pierro, G. Tempesta, Am. Mineral. 92 (2007), p. 403.

[4] H. Ott, Ztschr. f. Krist 61 (1925), p. 515.

[5] https://en.wikipedia.org/wiki/Polymorphs_of_silicon_carbide.

[6] This work is supported by the Natural Science Foundation of China under grant No. 51071125, and Major Project of Natural Science Foundation of Jiangxi Province under grant No. 20161ACB20010. Dr. Gert Nolze is thanked for kindly supplying the experimental EBSD patterns.
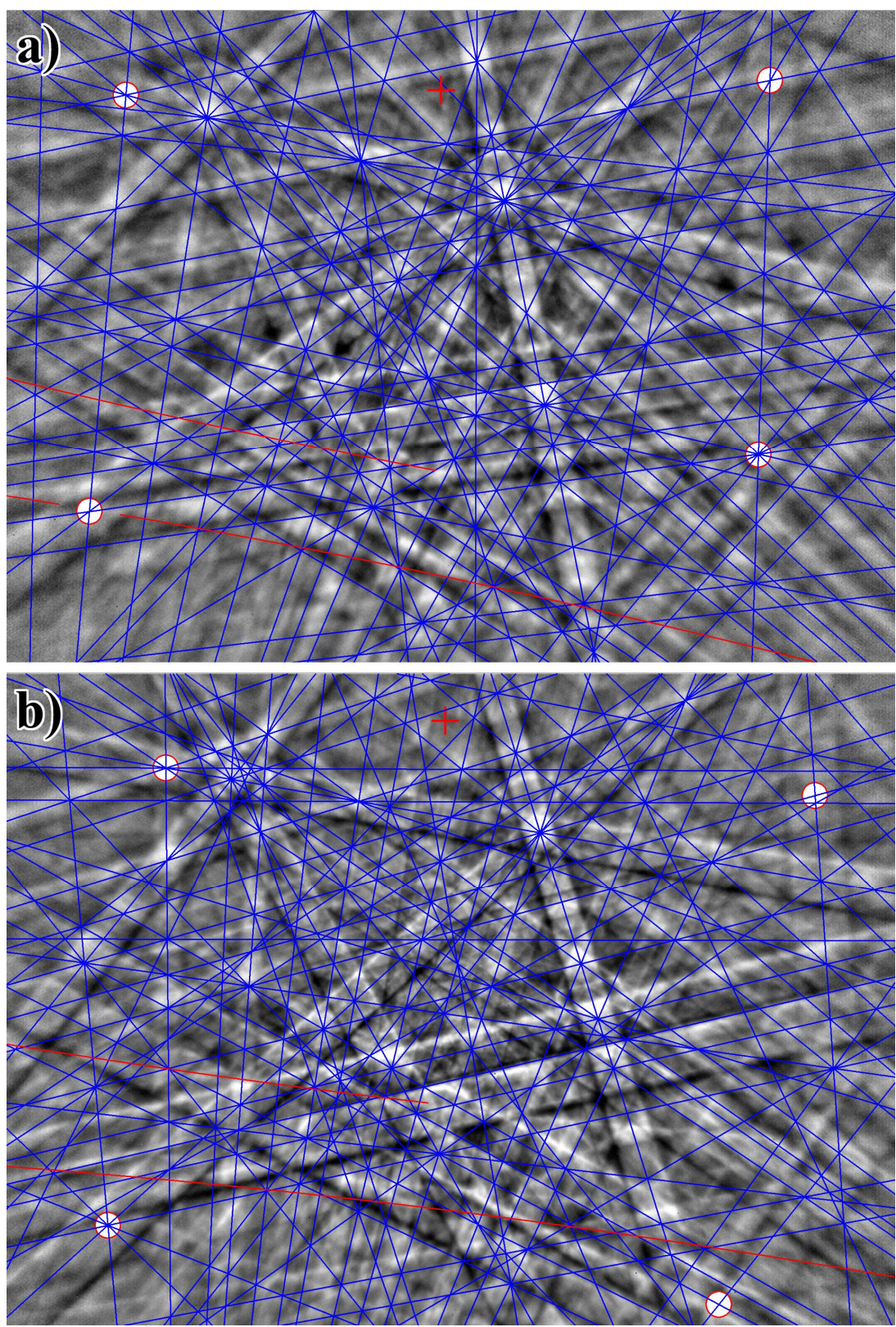

Figure 1. Experimentally collected EBSD patterns $(\mathrm{HT}=20 \mathrm{kV})$ of $\mathrm{SiC}$ crystals. The cross indicates the pattern center. a) Hexagonal Bravais lattice, b) Rhombohedral Bravais lattice. 